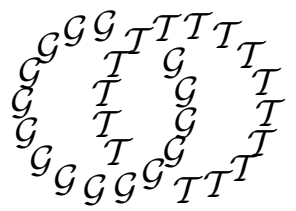

\title{
Reidemeister-Turaev torsion modulo one of rational homology three-spheres
}

\author{
Florian Deloup \\ GWÉnaËL Massuyeau
}

Laboratoire Emile Picard, UMR 5580 CNRS/Univ. Paul Sabatier 118 route de Narbonne, 31062 Toulouse Cedex 04, France

and

Laboratoire Jean Leray, UMR 6629 CNRS/Univ. de Nantes

2 rue de la Houssinière, BP 92208, 44322 Nantes Cedex 03, France

Email: deloup@picard.ups-tlse.fr and massuyea@math.univ-nantes.fr

\begin{abstract}
Given an oriented rational homology 3 -sphere $M$, it is known how to associate to any $\operatorname{Spin}^{c}$-structure $\sigma$ on $M$ two quadratic functions over the linking pairing. One quadratic function is derived from the reduction modulo 1 of the Reidemeister-Turaev torsion of $(M, \sigma)$, while the other one can be defined using the intersection pairing of an appropriate compact oriented 4-manifold with boundary $M$.

In this paper, using surgery presentations of the manifold $M$, we prove that those two quadratic functions coincide. Our proof relies on the comparison between two distinct combinatorial descriptions of $\operatorname{Spin}^{c}$-structures on $M$ : Turaev's charges vs Chern vectors.
\end{abstract}

\section{AMS Classification numbers Primary: 57M27}

Secondary: 57Q10, 57R15

Keywords: Rational homology 3-sphere, Reidemeister torsion, complex spin structure, quadratic function

Proposed: Robion Kirby

Seconded: Walter Neumann, Cameron Gordon
Received: 1 January 2003

Revised: 3 October 2003 


\section{Introduction and statement of the result}

\section{$1.1 \quad$ Introduction}

Any closed oriented 3-manifold $M$ can be equipped with a complex spin structure, or Spin ${ }^{c}$-structure. While they seem to have been originally introduced in the '50s and '60s [5], in the framework of Dirac operators and $K$-theory [8], the revival of interest in $\operatorname{Spin}^{c}$-structures over the last decade is certainly due to symplectic geometry and Seiberg-Witten invariants of 4-manifolds. For a general introduction to $\mathrm{Spin}^{c}$-structures, the reader is referred to [8]. It was observed somewhat more recently [16] that, in dimension 3, Spin ${ }^{c}$-structures have a simple and natural interpretation: any $\operatorname{Spin}^{c}$-structure on a closed oriented 3-manifold $M$ can be represented by a nowhere vanishing vector field on $M$. This enabled Turaev to reinterpret a topological invariant of Euler structures on 3-manifolds, which he had introduced earlier, as an invariant of $\mathrm{Spin}^{c}$-structures. Since this invariant is a refinement of the Reidemeister torsion, we call this invariant the Reidemeister-Turaev torsion.

We will be interested in the restriction of this invariant to the class of rational homology 3-spheres. Our work is motivated by and based on two observations.

- On the one hand, there is the following special feature of the ReidemeisterTuraev torsion $\tau_{M, \sigma}$ of an oriented rational homology 3-sphere $M$ with a Spin ${ }^{c}$-structure $\sigma$ : its reduction modulo 1 induces a quadratic function $q_{M, \sigma}$ over the linking pairing $\lambda_{M}[19]$.

- On the other hand, there is a canonical bijective correspondence, denoted by $\sigma \mapsto \phi_{M, \sigma}$, between $\operatorname{Spin}^{c}$-structures on $M$ and quadratic functions over the linking pairing $\lambda_{M}[10,4,2]$. The quadratic function $\phi_{M, \sigma}$ can be defined, extrinsically, using the intersection pairing of a compact oriented 4-manifold with boundary $M$ and first Betti number equal to zero.

Thus, the question naturally arises to compare the quadratic functions $q_{M, \sigma}$ and $\phi_{M, \sigma}$.

\subsection{Statement of the result}

Let us begin by developing the above two observations and fixing some notations.

The Reidemeister-Turaev torsion of a closed oriented 3-manifold equipped with a Spin ${ }^{c}-$ structure is a fundamental topological invariant. A concise and almost 
self-contained introduction is [14]. A broader introduction is [17], while the monographs $[11,19]$ contain the most recent developments. We give here a succinct presentation sufficient for our purpose.

Let $M$ be a connected oriented 3-manifold, compact without boundary. All homology and cohomology groups will be with integral coefficients unless explicity stated otherwise. We set $H=H_{1}(M)$, the first homology group, written multiplicatively. Let $Q(H)$ denote the classical ring of fractions of the group ring $\mathbb{Z}[H]$. The maximal Abelian Reidemeister torsion $\tau(M)$ of $M$ is an element in $Q(H)$ defined up to multiplication by an element of $\pm H \subset Q(H)$. This invariant, defined in [13], can be thought of as a generalization of the Alexander polynomial. Next, its indeterminacy in $\pm H$ can be disposed of by specifying two extra structures: a homology orientation of $M$ and an Euler structure of $M$ (see [15]). On the one hand, using the intersection pairing, the choosen orientation of $M$ induces a canonical homology orientation. On the other hand, the Euler structures on $M$, defined as punctured homotopy classes of nowhere vanishing vector fields on $M$, are in canonical bijective correspondence with the Spin ${ }^{c}$-structures on $M[16]$. Therefore, if $(M, \sigma)$ is a connected closed Spin $^{c}$-manifold of dimension 3, one can define its Reidemeister-Turaev torsion

$$
\tau(M, \sigma) \in Q(H) .
$$

It has the following equivariance property:

$$
\forall h \in H, \quad h \cdot \tau(M, \sigma)=\tau(M, h \cdot \sigma) \in Q(H) .
$$

Here, the left hand side involves a multiplication in $Q(H)$ while, in the right hand side, $h \cdot \sigma$ involves the free and transitive action of $H^{2}(M)$ (or $H_{1}(M)$ via Poincaré duality) on the set $\operatorname{Spin}^{c}(M)$ : see, eg, [8].

Now and throughout the paper, we assume that $M$ is an oriented rational homology 3-sphere, ie, we suppose that

$$
H_{*}(M ; \mathbb{Q})=H_{*}\left(\mathbf{S}^{3} ; \mathbb{Q}\right) .
$$

Then $H$ is finite and $Q(H)=\mathbb{Q}[H]$. Hence $\tau(M, \sigma)$ determines a function $\tau_{\sigma}: H \rightarrow \mathbb{Q}$ such that

$$
\tau(M, \sigma)=\sum_{h \in H} \tau_{\sigma}(h) \cdot h \in \mathbb{Q}[H] .
$$

It has been proved in [16, Theorem 4.3.1] that the modulo 1 reduction of the function $\tau_{\sigma}$ satisfies the property that

$$
\forall h_{1}, h_{2} \in H, \quad \tau_{\sigma}\left(h_{1} h_{2}\right)-\tau_{\sigma}\left(h_{1}\right)-\tau_{\sigma}\left(h_{2}\right)+\tau_{\sigma}(1)=-\lambda_{M}\left(h_{1}, h_{2}\right) \bmod 1 .
$$


Here, $\lambda_{M}: H \times H \rightarrow \mathbb{Q} / \mathbb{Z}$ denotes the linking pairing of $M$ : this is a symmetric nondegenerate bilinear pairing, which gives partial information on the way knots are linked in the manifold $M$ [12]. It immediately follows from (1.2) that

$$
\forall h \in H, \quad \tau_{\sigma}(h)=\tau_{\sigma}(1)-q_{M, \sigma}\left(h^{-1}\right) \bmod 1,
$$

where $q_{M, \sigma}$ is a quadratic function over the linking pairing $\lambda_{M}$, in the sense that it satisfies the following property:

$$
\forall h, k \in H, \quad q_{M, \sigma}(h k)-q_{M, \sigma}(h)-q_{M, \sigma}(k)=\lambda_{M}(h, k) .
$$

It is also easily seen from (1.1) and (1.2) that

$$
\forall h \in H, \quad q_{M, h \cdot \sigma}=q_{M, \sigma}+\lambda_{M}(h,-) .
$$

This equation suggests to define the following free transitive action of the group $H$ on the set $\operatorname{Quad}\left(\lambda_{M}\right)$ of quadratic functions over $\lambda_{M}$ :

$$
H \times \operatorname{Quad}\left(\lambda_{M}\right) \rightarrow \operatorname{Quad}\left(\lambda_{M}\right),(h, q) \mapsto h \cdot q
$$

where

$$
\forall x \in H,(h \cdot q)(x)=q(x)+\lambda_{M}(h, x) .
$$

On the other hand, it is known [10, 4, 2] (see [3] for arbitrary closed oriented 3 -manifolds) how to define another bijective $H$-equivariant correspondence

$$
\operatorname{Spin}^{c}(M) \rightarrow \operatorname{Quad}\left(\lambda_{M}\right), \sigma \mapsto \phi_{M, \sigma} .
$$

This map is defined combinatorially, starting from a surgery presentation of the manifold $M$ and using its linking matrix. (The detailed construction will be recalled in subsection 2.4.)

Theorem For any oriented rational homology 3-sphere $M$ equipped with a Spin ${ }^{c}$-structure $\sigma$, the quadratic functions $q_{M, \sigma}$ and $\phi_{M, \sigma}$ are equal.

In his monograph [11], Nicolaescu has proved the same result, with an analytic proof based on the connection between the Reidemeister-Turaev torsion and the Seiberg-Witten invariant. Our proof is combinatorial and purely topological. A surgery presentation of $M$ provides two combinatorial descriptions of $\operatorname{Spin}^{c}$ structures on $M$. One description (called charges) is defined by Turaev in [18] in terms of the complement in $\mathbf{S}^{3}$ of the framed surgery link, and is used there to compute $\tau(M, \sigma)$. Another description (called Chern vectors) relies on the 4-manifold with boundary $M$ associated to the surgery presentation, and is well suited for the computation of $\phi_{M, \sigma}$. Our main contribution consists in comparing those two descriptions of $\mathrm{Spin}^{c}$-structures.

Before going into the proof of the Theorem, let us discuss the following immediate consequence. 
Corollary The quadratic function $\phi_{M, \sigma}$ is determined by $\tau(M, \sigma) \bmod 1$.

We claim that the converse of the Corollary does not hold. To justify this, define the "constant"

$$
c_{\sigma}=\tau_{\sigma}(1) \bmod 1
$$

From (1.1), we obtain that

$$
\forall h \in H, \quad c_{h \cdot \sigma}=c_{\sigma}-\phi_{M, \sigma}(h) .
$$

Let also $d_{\sigma} \in \mathbb{R} / \mathbb{Z}$ be such that

$$
\exp \left(2 i \pi d_{\sigma}\right)=\frac{1}{\sqrt{|H|}} \cdot \sum_{x \in H} \exp \left(2 i \pi \phi_{M, \sigma}(x)\right) \in \mathbb{C} .
$$

Since $\phi_{M, \sigma}$ is nondegenerate, the Gauss sum on the right hand side is wellknown to be a complex number of modulus 1 . It can also be proved that $d_{\sigma} \in \mathbb{Q} / \mathbb{Z}$. Observe that

$$
d_{h \cdot \sigma}=d_{\sigma}-\phi_{M, \sigma}(h) .
$$

As an immediate consequence of (1.4) and (1.5), we obtain the following

Proposition The number $c(M)=c_{\sigma}-d_{\sigma} \in \mathbb{Q} / \mathbb{Z}$ is a topological invariant of the oriented rational homology 3-sphere $M$.

Explicit computations can be performed on the lens spaces. For instance, we find that $8 c(L(7,1))=3 / 7 \neq 2 / 7=8 c(L(7,2))$; since $L(7,1)$ and $L(7,2)$ have isomorphic linking pairings, we deduce that $c(M)$ can not be computed from $\phi_{M, \sigma}$.

It is not difficult to verify that $c(M)$ is additive under connected sums, vanishes if $M$ is an integer homology 3 -sphere and changes sign when the orientation of $M$ is reversed. Let $\lambda(M) \in \mathbb{Q}$ denote the Casson-Walker invariant of $M$ in Lescop's normalization [9]. We ask the following

Question Does the invariant $c(M) \in \mathbb{Q} / \mathbb{Z}$ coincide with $-\lambda(M) /|H| \bmod 1$ ?

Acknowledgements The first author is an EU Marie Curie Research Fellow (HPMF 2001-01174) at the Einstein Institute of Mathematics, the Hebrew University of Jerusalem. 


\section{Chern vectors and charges}

This section contains preliminary material for the proof of the Theorem (Section 3 ). The heart of this section is devoted to the presentation of two equivalent, but distinct, combinatorial descriptions of complex spin structures on $M$. The proof of this equivalence will be given in Section 3. Even though we shall not need it, note that subsections 2.1, 2.2 and 2.3 are valid for any closed oriented connected 3-manifold (ie, with arbitrary first Betti number).

As a convention, boundaries of oriented manifolds will be always given orientation by the "outward normal vector first" rule.

\section{$2.1 \quad$ Surgery presentation}

In this paragraph and throughout Section 2, we fix an ordered oriented framed $n$-component link $L$ in $\mathbf{S}^{3}$, such that the oriented 3 -manifold $V_{L}$ obtained from $\mathbf{S}^{3}$ by surgery along $L$ is diffeomorphic to our oriented rational homology 3-sphere $M$.

Let $b_{i j}=\operatorname{lk}_{\mathbf{S}^{3}}\left(L_{i}, L_{j}\right)$ for all $1 \leq i \neq j \leq n$, and let $b_{i i}$ be the framing number of $L_{i}$ for all $1 \leq i \leq n$. We denote by $B_{L}=\left(b_{i j}\right)_{i, j=1, \ldots, n}$ the linking matrix of $L$ in $\mathbf{S}^{3}$. We also denote by $W_{L}$ the trace of the surgery. In other words,

$$
M=V_{L}=\partial W_{L} \quad \text { with } \quad W_{L}=\mathbf{D}^{4} \cup \bigcup_{i=1}^{n}\left(\mathbf{D}^{2} \times \mathbf{D}^{2}\right)_{i},
$$

where the 2-handle $\left(\mathbf{D}^{2} \times \mathbf{D}^{2}\right)_{i}$ is attached by embedding $-\left(\mathbf{S}^{1} \times \mathbf{D}^{2}\right)_{i}$ into $\mathbf{S}^{3}=\partial \mathbf{D}^{4}$ in accordance with the specified framing and orientation of $L_{i}$. The group $H_{2}\left(W_{L}\right)$ is free Abelian of rank $n$. It is given the preferred basis $\left(\left[S_{1}\right], \ldots,\left[S_{n}\right]\right)$. Here, the closed surface $S_{i}$ is taken to be

$$
S_{i}=\left(\mathbf{D}^{2} \times 0\right)_{i} \cup\left(-\Sigma_{i}\right),
$$

where $\Sigma_{i}$ is a Seifert surface for $L_{i}$ in $\mathbf{S}^{3}$ which has been pushed into the interior of $\mathbf{D}^{4}$ as shown in Figure 2.1. Also, $H^{2}\left(W_{L}\right)$ will be identified with $\operatorname{Hom}\left(H_{2}\left(W_{L}\right), \mathbb{Z}\right)$ by Kronecker evaluation, and will be given the dual basis. Note that the matrix of the intersection pairing $\bullet: H_{2}\left(W_{L}\right) \times H_{2}\left(W_{L}\right) \rightarrow \mathbb{Z}$ relatively to the preferred basis of $H_{2}\left(W_{L}\right)$ is $B_{L}$. 


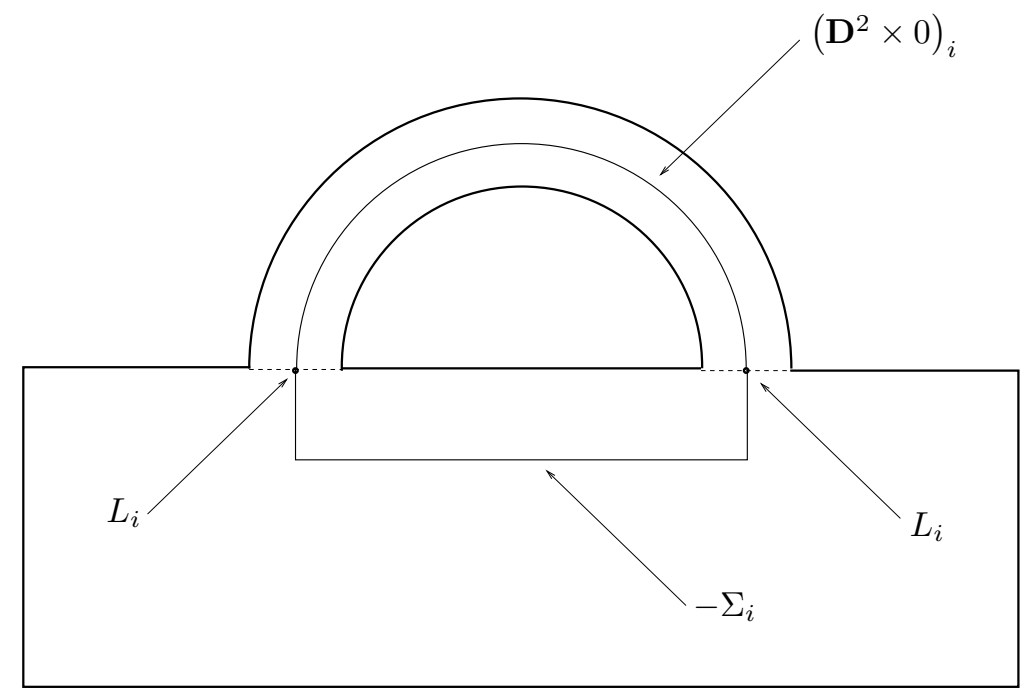

Figure 2.1: The preferred basis of $H_{2}\left(W_{L}\right)$

\subsection{Chern vectors}

We define the set of Chern vectors (associated to the link $L$ ) to be

$$
\tilde{\mathcal{V}}_{L}=\left\{s=\left(s_{i}\right)_{i=1}^{n} \in \mathbb{Z}^{n}: \forall i=1, \ldots, n, s_{i} \equiv b_{i i} \bmod 2\right\} .
$$

Set $\mathcal{V}_{L}=\frac{\tilde{\mathcal{V}}_{L}}{2 \cdot \operatorname{Im} B_{L}}$. A basic result of [3] (where the reader is referred to for full details) asserts that

$$
\operatorname{Spin}^{c}\left(V_{L}\right) \simeq \mathcal{V}_{L}
$$

This is our first combinatorial description of $\operatorname{Spin}^{c}$-structures on $V_{L}$, which we now recall briefly. Let $\sigma \in \operatorname{Spin}^{c}\left(V_{L}\right)$. Extend $\sigma$ to a $\operatorname{Spin}^{c}$-structure $\tilde{\sigma} \in \operatorname{Spin}^{c}\left(W_{L}\right)$. Thus the Chern class $c(\tilde{\sigma}) \in H^{2}\left(W_{L}\right) \simeq \operatorname{Hom}\left(H_{2}\left(W_{L}\right), \mathbb{Z}\right)$ is given by an element in $\mathbb{Z}^{n}$ (according to the basis dual to the preferred basis). The isomorphism (2.1) is induced by the map $\sigma \mapsto c(\tilde{\sigma})$.

\subsection{Charges}

Charges were introduced by Turaev in [18], as a combinatorial description of Euler structures. We give a brief description.

The set of charges (associated to the link $L$ ) is defined to be 


$$
\tilde{\mathcal{C}}_{L}=\left\{k=\left(k_{i}\right)_{i=1}^{n} \in \mathbb{Z}^{n}: \forall i=1, \ldots, n, k_{i} \equiv 1+\sum_{1 \leq j \leq n, j \neq i} b_{i j} \bmod 2\right\} .
$$

Set $\mathcal{C}_{L}=\frac{\tilde{\mathcal{C}}_{L}}{2 \cdot \operatorname{Im} B_{L}}$. We shall recall below that

$$
\operatorname{Spin}^{c}\left(V_{L}\right) \simeq \mathcal{C}_{L}
$$

We can alternatively view $V_{L}$, without reference to $W_{L}$, as

$$
V_{L}=\mathbf{E} \cup \bigcup_{i=1}^{n} Z_{i}
$$

where $\mathbf{E}$ denotes the exterior of a tubular neighborhood of $L$ in $\mathbf{S}^{3}$ and $Z_{i}$ is a (reglued) solid torus, homeomorphic to $\mathbf{S}^{1} \times \mathbf{D}^{2}$. A solid torus $Z$ is said to be directed when its core is oriented. We direct the solid torus $Z_{j}$ in the following way: we denote by $m_{j} \subset \mathbf{E}$ the meridian of $L_{j}$ which is oriented so that $\operatorname{lk}_{\mathbf{S}^{3}}\left(m_{j}, L_{j}\right)=+1$, and we require the oriented core of $Z_{j}$ to be isotopic in $V_{L}$ to $m_{j}$.

In general, let $N$ be a compact oriented 3 -manifold with boundary $\partial N$ endowed, this time, with a Spin-structure $\sigma$. There is a well-defined set of

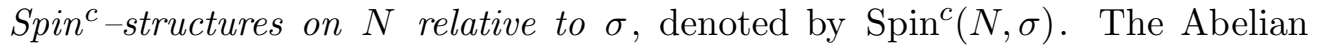
group $H^{2}(N, \partial N)$ acts freely and transitively on $\operatorname{Spin}^{c}(N, \sigma)$. Also, there is a Chern class map

$$
c: \operatorname{Spin}^{c}(N, \sigma) \rightarrow H^{2}(N, \partial N)
$$

which is affine over the square map (where $H^{2}(N, \partial N)$ is written multiplicatively). For details about relative $\operatorname{Spin}^{c}$-structures and their gluings, see [3].

The torus $\mathbf{S}^{1} \times \mathbf{S}^{1}$ has a canonical Spin-structure $\sigma^{0}$, which is induced by its Lie group structure. Hence $\partial \mathbf{E}$ can be endowed with a distinguished Spinstructure, which is denoted by $\cup_{i=1}^{n} \sigma^{0}$. A directed solid torus $Z$ has a distinguished Spin ${ }^{c}$-structure relative to the canonical Spin-structure $\sigma^{0}$ on $\partial Z$ : this is the one whose Chern class is Poincare dual to the opposite of the oriented core of $Z$. Hence by gluing any $\operatorname{Spin}^{c}$-structure on $\mathbf{E}$ relative to $\cup_{i=1}^{n} \sigma^{0}$ to the distinguished relative $\operatorname{Spin}^{c}{ }^{c}$ structures on the directed solid tori $Z_{j}$ 's, we define a map

$$
g: \operatorname{Spin}^{c}\left(\mathbf{E}, \cup_{i=1}^{n} \sigma^{0}\right) \rightarrow \operatorname{Spin}^{c}\left(V_{L}\right) .
$$

This map $g$ is affine, via the Poincaré duality isomorphisms $P: H_{1}(\mathbf{E}) \rightarrow$ $H^{2}(\mathbf{E}, \partial \mathbf{E})$ and $P: H_{1}\left(V_{L}\right) \rightarrow H^{2}\left(V_{L}\right)$, over the natural inclusion homomorphism $H_{1}(\mathbf{E}) \rightarrow H_{1}\left(V_{L}\right)$. In particular, $g$ is onto. 
Another useful general fact is that the Chern class $c(\alpha)$ of a $\operatorname{Spin}^{c}$-structure $\alpha$ relative to a Spin-structure on the boundary has a nice explicit expression modulo 2, which we briefly explain. Let $S$ be a closed oriented surface. Denote by $\operatorname{Quad}(S)$ the set of quadratic functions over the mod 2 intersection pairing of $S$. Hence, an element $q \in \operatorname{Quad}(S)$ is a map $q: H_{1}\left(S ; \mathbb{Z}_{2}\right) \rightarrow \mathbb{Z}_{2}$ such that $q(x+y)-q(x)-q(y)=x \bullet y$ for all $x, y \in H_{1}\left(S ; \mathbb{Z}_{2}\right)$, where $\bullet$ denotes the mod 2 intersection pairing. The Atiyah-Johnson correspondence $[1,6]$ is a bijective $H_{1}\left(S, \mathbb{Z}_{2}\right)$-equivariant map

$$
J: \operatorname{Spin}(S) \rightarrow \operatorname{Quad}(S), \sigma \mapsto J_{\sigma} .
$$

Here, the function $J_{\sigma}$ is defined, for any simple oriented closed curve $\gamma$, by $J_{\sigma}([\gamma])=1$ or 0 according to whether $\left(\gamma,\left.\sigma\right|_{\gamma}\right)$ is homotopic to $\mathbf{S}^{1}$ with the Spin-structure induced from the Lie group structure or not [7, pages 35-36].

Lemma 2.1 (See [3]) Let $N$ be a compact oriented 3-manifold with boundary, $\sigma \in \operatorname{Spin}(\partial N)$ and $\alpha \in \operatorname{Spin}^{c}(N, \sigma)$. Then

$$
\forall y \in H_{2}(N, \partial N), \quad\langle c(\alpha), y\rangle \equiv J_{\sigma}\left(\partial_{*}(y)\right) \bmod 2,
$$

where $\langle\cdot, \cdot\rangle$ denotes Kronecker evaluation, and where $\partial_{*}: H_{2}(N, \partial N) \rightarrow H_{1}(\partial N)$ is the connecting homomorphism of the pair $(N, \partial N)$.

A canonical bijection between $\operatorname{Spin}^{c}\left(\mathbf{E}, \cup_{i=1}^{n} \sigma^{0}\right)$ and $\tilde{\mathcal{C}}_{L}$ can be defined in the following way: for any $\alpha \in \operatorname{Spin}^{c}\left(\mathbf{E}, \cup_{i=1}^{n} \sigma^{0}\right)$, calculate $P^{-1} c(\alpha) \in H_{1}(\mathbf{E})$ and identify $H_{1}(\mathbf{E})$ with $\mathbb{Z}^{n}$ taking the meridians $\left(\left[m_{1}\right], \ldots,\left[m_{n}\right]\right)$ as a basis; it is a consequence of Lemma 2.1 that the multi-integer we obtain is actually a charge on $L$. Thus, since $g$ is surjective and since $\operatorname{Ker}\left(H_{1}(\mathbf{E}) \rightarrow H_{1}\left(V_{L}\right)\right)$ is generated by the $n$ characteristic curves of the surgery, it follows that the map $g$ induces a bijection

$$
\frac{\tilde{\mathcal{C}}_{L}}{2 \cdot \operatorname{Im} B_{L}} \rightarrow \operatorname{Spin}^{c}\left(V_{L}\right)
$$

as claimed.

\subsection{The quadratic function $\phi_{M, \sigma}$}

In this paragraph, we recall how to compute the quadratic function $\phi_{M, \sigma}$ $[10,4,2]$ from the surgery presentation $L$ for $M$ and a Chern vector $s \in \mathbb{Z}^{n}$ representing $\sigma \in \operatorname{Spin}^{c}(M)$. By the homology exact sequence associated to the pair $\left(W_{L}, V_{L}\right)$, the choice of the preferred basis for $H_{2}\left(W_{L}\right)$ induces an identification

$$
H \simeq \text { Coker } B_{L}=\mathbb{Z}^{n} / \operatorname{Im} B_{L}
$$


Let $x \in H$ and let $X \in \mathbb{Z}^{n}$ be a representative of $x$ by (2.3). We have

$$
\phi_{M, \sigma}(x)=-\frac{1}{2}\left(X^{\mathrm{T}} \cdot B_{L}^{-1} \cdot X+X^{\mathrm{T}} \cdot B_{L}^{-1} \cdot s\right) \bmod 1 .
$$

Example 2.2 Suppose that the surgery link $L$ is algebraically split (ie, $B_{L}$ is diagonal). As before, denote by $m_{i}$ the meridian of $L_{i}$ oriented so that $\operatorname{lk}_{\mathbf{S}^{3}}\left(L_{i}, m_{i}\right)=+1$ and let $\left[m_{i}\right] \in H$ be its homology class in $M$. It follows from (2.3) and the orientation convention that

$$
\phi_{M, \sigma}\left(\left[m_{i}\right]\right)=-\frac{1}{2 b_{i i}}\left(1-s_{i}\right) \bmod 1 .
$$

\section{Proof of the Theorem}

A technical difficulty lies in the computation of $q_{M, \sigma}$ from the torsion $\tau(M, \sigma)$. Fortunately, $\tau(M, \sigma)$ can be computed from a surgery presentation of $M$ and a charge representing $\sigma$ (see [18] or [19]). In the previous section, we computed $\phi_{M, \sigma}$ from a surgery presentation of $M$ and a Chern vector representing $\sigma$. Thus, the proof consists in two steps: 1. compare charges to Chern vectors (there must be a bijective correspondence between them); 2. compare $q_{M, \sigma}$ to $\phi_{M, \sigma}$ using surgery presentations.

We shall use the notations of the previous section. In particular, we have fixed an ordered oriented framed $n$-component link $L$ in $\mathbf{S}^{3}$, such that the oriented 3-manifold $V_{L}$ obtained by surgery along $L$ is diffeomorphic to our oriented rational homology 3-sphere $M$.

The comparison of the two combinatorial descriptions of $\operatorname{Spin}^{c}\left(V_{L}\right)$ is contained in the following

Claim 3.1 If $\sigma \in \operatorname{Spin}^{c}\left(V_{L}\right)$ corresponds to $[k] \in \mathcal{C}_{L}$, then $\sigma$ corresponds to $[s] \in \mathcal{V}_{L}$, where

$$
\forall j \in\{1, \ldots, n\}, \quad s_{j}=1-k_{j}+\sum_{i=1}^{n} b_{i j} .
$$

Remark 3.2 Claim 3.1 is true for any closed oriented connected 3-manifold (ie, with arbitrary first Betti number). 


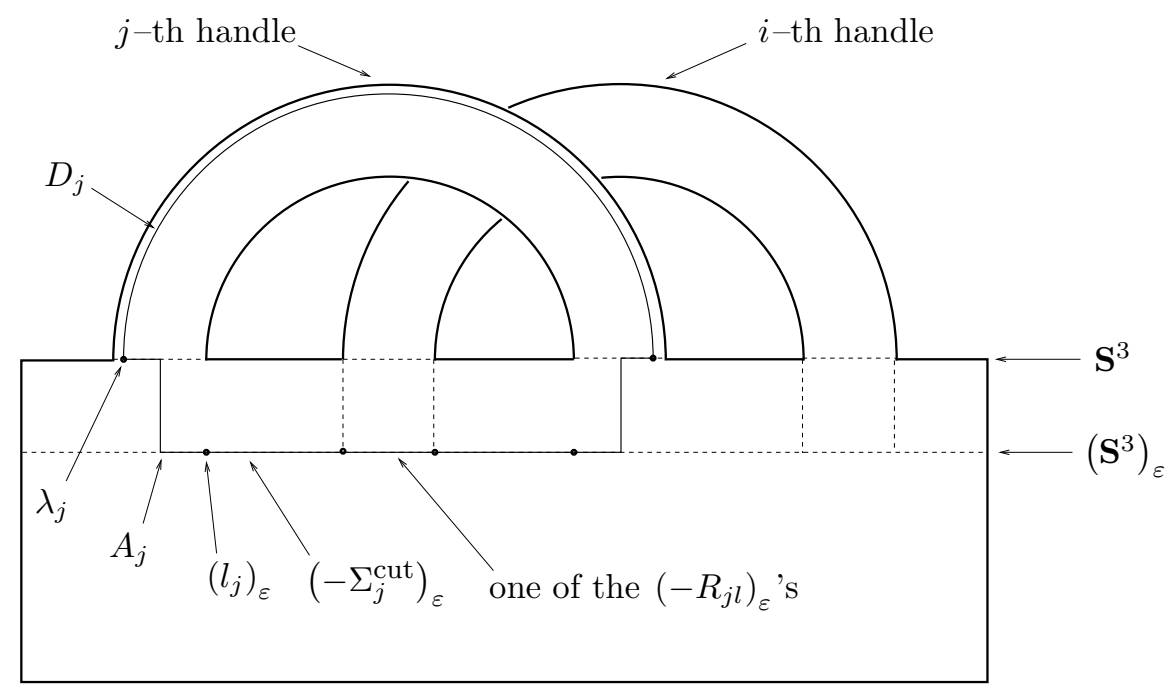

Figure 3.1: A decomposition of the surface $S_{j}$

Proof of the Claim 3.1 We denote by $\sigma_{2}$ the distinguished relative Spin ${ }^{c}-$ structure in $\operatorname{Spin}^{c}\left(\cup_{j=1}^{n} Z_{j}, \cup_{j=1}^{n} \sigma^{0}\right)$. Let also $\sigma_{1} \in \operatorname{Spin}^{c}\left(\mathbf{E}, \cup_{j=1}^{n} \sigma^{0}\right)$ be such that

$$
\sigma=\sigma_{1} \cup \sigma_{2} \in \operatorname{Spin}^{c}\left(V_{L}\right) .
$$

Pick an extension $\tilde{\sigma}$ of $\sigma$ to $W_{L}$ and let $\xi$ be the isomorphism class of $U(1)-$ principal bundles determined by $\tilde{\sigma} \in \operatorname{Spin}^{c}\left(W_{L}\right)$. On the one hand, the first Chern class $c_{1}(\xi)$ of $\xi$, when expressed in the preferred basis $\left(\left[S_{j}\right]^{*}\right)_{j=1}^{n}$ of $H^{2}\left(W_{L}\right) \simeq \operatorname{Hom}\left(H_{2}\left(W_{L}\right), \mathbb{Z}\right)$, gives a multi-integer $s \in \mathbb{Z}^{n}$; then $[s] \in \mathcal{V}_{L}$ corresponds to $\sigma$. On the other hand, the Poincaré dual to the relative Chern class of $\sigma_{1} \in \operatorname{Spin}^{c}\left(\mathbf{E}, \cup_{j=1}^{n} \sigma^{0}\right)$, when expressed in the preferred basis $\left(\left[m_{j}\right]\right)_{j=1}^{n}$ of $H_{1}(\mathbf{E})$, gives a multi-integer $k \in \mathbb{Z}^{n}$; then $[k] \in \mathcal{C}_{L}$ corresponds to $\sigma$. Thus, proving that those specific integers $k$ and $s$ verify (3.1) modulo $2 \cdot \operatorname{Im} B_{L}$ will be enough.

In the sequel we denote by $\left(\mathbf{S}^{3}\right)_{\varepsilon}$ a collar push-off of $\mathbf{S}^{3}=\partial \mathbf{D}^{4}$ in the interior of $\mathbf{D}^{4}$. The surface $S_{j}$ can be decomposed (up to isotopy) in $W_{L}$ as

$$
S_{j}=D_{j} \cup A_{j} \cup\left(-\Sigma_{j}^{\mathrm{cut}}\right)_{\varepsilon} \cup \bigcup_{l}\left(-R_{j l}\right)_{\varepsilon}
$$

where the subsurfaces, illustrated on Figure 3.1, are defined as follows:

- $D_{j}$ is a meridian disc of $Z_{j}$ such that $\partial D_{j}$ is the characteristic curve $\lambda_{j}$ of the $j$-th surgery; 
- $A_{j}$ is the annulus of an isotopy of $-\lambda_{j}$ to $L_{j}$, union the annulus of an isotopy of $-L_{j}$ to $\left(L_{j}\right)_{\varepsilon}$, union the annulus of an isotopy of $\left(-L_{j}\right)_{\varepsilon}$ to $\left(l_{j}\right)_{\varepsilon}$, where $l_{j}$ denotes the preferred parallel of $L_{j}$ in $\mathbf{S}^{3}\left(\mathrm{ie}, \mathrm{lk}_{\mathbf{S}^{3}}\left(l_{j}, L_{j}\right)=\right.$ $0)$;

- $\Sigma_{j}$ is a Seifert surface for $l_{j}$ in $\mathbf{S}^{3}$ disjoint from $L_{j}$ and in transverse position with the $L_{i}$ 's $(i \neq j)$. For each intersection point $x_{l}$ between $\Sigma_{j}$ and a $L_{i}$, remove a small disc $R_{j l}$ so that $\Sigma_{j}=\Sigma_{j}^{\text {cut }} \cup \bigcup_{l} R_{j l}$.

By definition of $s$, we have $s_{j}=\left\langle c_{1}(\xi),\left[S_{j}\right]\right\rangle=\left\langle c_{1}\left(\left.p\right|_{S_{j}}\right),\left[S_{j}\right]\right\rangle$ where $p$ is representative for $\xi$ and where $c_{1}\left(\left.p\right|_{S_{j}}\right) \in H^{2}\left(S_{j}\right)$ is the obstruction to trivialize $p$ over $S_{j}$. So $P^{-1} c_{1}\left(\left.p\right|_{S_{j}}\right)=s_{j} \cdot[\mathrm{pt}] \in H_{0}\left(S_{j}\right)$. Let tr be a trivialization of $p$ on $\partial \mathbf{E}$ and let $\operatorname{tr}_{\varepsilon}$ be the corresponding trivialization of $p$ on $(\partial \mathbf{E})_{\varepsilon}$. A classical argument (calculus of obstructions in compact oriented manifolds by means of Poincaré dualities) leads to the equality

$$
\begin{aligned}
H_{0}\left(S_{j}\right) \ni P^{-1} c_{1}\left(\left.p\right|_{S_{j}}\right)= & i_{*} P^{-1} c_{1}\left(\left.p\right|_{D_{j}},\left.\operatorname{tr}\right|_{\lambda_{j}}\right) \\
& +i_{*} P^{-1} c_{1}\left(\left.p\right|_{A_{j}},\left.\left.\operatorname{tr}\right|_{-\lambda_{j}} \cup \operatorname{tr}_{\varepsilon}\right|_{\left(l_{j}\right)_{\varepsilon}}\right) \\
& -i_{*} P^{-1} c_{1}\left(\left.p\right|_{\left.\left(\Sigma_{j}^{\text {cut }}\right)_{\varepsilon},\left.\operatorname{tr}_{\varepsilon}\right|_{\left(\partial \Sigma_{j}^{\text {cut }}\right)_{\varepsilon}}\right)}\right. \\
& -\sum_{l} i_{*} P^{-1} c_{1}\left(\left.p\right|_{\left(R_{j l}\right)_{\varepsilon}},\left.\operatorname{tr}_{\varepsilon}\right|_{\left(\partial R_{j l}\right)_{\varepsilon}}\right),
\end{aligned}
$$

where $P$ denotes a Poincaré duality isomorphism for the appropriate surface $\left(D_{j}, A_{j}, \Sigma_{j}^{\text {cut }}\right.$ or $\left.R_{j l}\right)$. For an appropriate choice of $p$ in the class $\xi$ and for an appropriate choice of tr, we have

$$
\begin{aligned}
c_{1}\left(\left.p\right|_{\mathbf{E}}, \mathrm{tr}\right) & =c\left(\sigma_{1}\right) \in H^{2}(\mathbf{E}, \partial \mathbf{E}) \\
c_{1}\left(\left.p\right|_{\cup_{j} Z_{j}}, \mathrm{tr}\right) & =c\left(\sigma_{2}\right) \in H^{2}\left(\cup_{j} Z_{j}, \cup_{j} \partial Z_{j}\right) \\
c_{1}\left(\left.p\right|_{\mathrm{N}(L)}, \mathrm{tr}\right) & =c\left(\sigma_{3}\right) \in H^{2}(\mathrm{~N}(L), \partial \mathrm{N}(L))
\end{aligned}
$$

where, in this last requirement, $\mathrm{N}(L)$ is a tubular neighborhood of $L$ in $\mathbf{S}^{3}$ and $\sigma_{3}$ is an arbitrary element of $\operatorname{Spin}^{c}\left(\mathrm{~N}(L), \cup_{j} \sigma^{0}\right)$. For such choices, we now compute separately each term of the right hand side of (3.2).

(1) The first term is of the form $d_{j} \cdot[\mathrm{pt}]$. Here

$$
d_{j}=\left\langle c\left(\sigma_{2}\right),\left[D_{j}\right]\right\rangle=-\left(\text { oriented core of } Z_{j}\right) \bullet\left[D_{j}\right]=+1,
$$

where the intersection is taken in $Z_{j}$. (Note that $Z_{j}=\left(\mathbf{D}^{2} \times \mathbf{S}^{1}\right)_{j}$ if we denote by $\left(\mathbf{D}^{2} \times \mathbf{D}^{2}\right)_{j}$ the 2 -handle of $W_{L}$ corresponding to $L_{j}$, and be careful of the fact that the above specified oriented core of $Z_{j}$ is $\left.-\left(0 \times \mathbf{S}^{1}\right)_{j}.\right)$ 
(2) The second term is of the form $a_{j} \cdot[\mathrm{pt}]$. Here $a_{j}=\left\langle c\left(\sigma_{3}\right),\left[A_{j}\right]\right\rangle$ where $A_{j}$ is regarded as a relative $2-$ cycle in $(\mathrm{N}(L), \partial \mathrm{N}(L))$ once the collar has been squeezed. Since $\partial A_{j}$ is $-\lambda_{j} \cup l_{j},\left[A_{j}\right]$ is $-b_{j j}$ times the class of the meridian disc of $L_{j}$ (oriented so that its oriented boundary is $m_{j}$ ) in $H_{2}(\mathrm{~N}(L), \partial \mathrm{N}(L))$. Then, $a_{j}=-b_{j j} \cdot \rho_{j}$ where $\rho_{j}$ is defined to be

$$
\rho_{j}=\left\langle c\left(\sigma_{3}\right),\left[\text { meridian disc of } L_{j}\right]\right\rangle \in \mathbb{Z} .
$$

Note that $\rho_{j} \equiv J_{\sigma^{0}}\left(\left[m_{j}\right]\right) \equiv 1 \bmod 2$ (by the Atiyah-Johnson correspondence, see Lemma 2.1).

(3) The third term is $-g_{j} \cdot[\mathrm{pt}]$ where $g_{j}=\left\langle c\left(\sigma_{1}\right),\left[\Sigma_{j}^{\text {cut }}\right]\right\rangle$. But, that integer is equal to

$$
g_{j}=\left(P^{-1} c\left(\sigma_{1}\right)\right) \bullet\left[\Sigma_{j}^{\mathrm{cut}}\right]=\left(\sum_{i} k_{i}\left[m_{i}\right]\right) \bullet\left[\Sigma_{j}^{\mathrm{cut}}\right]=\sum_{i} k_{i} \delta_{i j}=k_{j}
$$

where the intersection is taken in $\mathbf{E}$.

(4) The fourth term is given by $-\sum_{l} r_{j l} \cdot[\mathrm{pt}]$. Here $r_{j l}=\left\langle c\left(\sigma_{3}\right),\left[R_{j l}\right]\right\rangle$. For each index $l$, denote by $i(l)$ the integer $i$ such that $x_{l}$ is an intersection point of $\Sigma_{j}$ with $L_{i}$, and denote by $\epsilon(l)$ the sign of the intersection point $x_{l}$. Then, from the definition of $\rho_{i}$ (given for the second term), we have $r_{j l}=\epsilon(l) \cdot \rho_{i(l)}$. Hence

$$
\sum_{l} r_{j l}=\sum_{\substack{i=1 \\ i \neq j}}^{n} b_{i j} \rho_{i}
$$

Putting those computations together, we obtain that (3.2) is equivalent to the identity

$$
\begin{aligned}
s_{j} & =d_{j}+a_{j}-g_{j}-\sum_{l} r_{j l} \\
& =1-b_{j j} \rho_{j}-k_{j}-\sum_{\substack{i=1 \\
i \neq j}}^{n} b_{i j} \rho_{i} \\
& =\left(1-k_{j}+\sum_{i=1}^{n} b_{i j}\right)-\sum_{i=1}^{n} b_{i j}\left(\rho_{i}+1\right) .
\end{aligned}
$$

The claim now follows from the fact that $\rho_{i} \equiv 1 \bmod 2$ for all $i=1, \ldots, n$.

We are now able to prove the Theorem. Assume first that $M$ is obtained by surgery along an algebraically split link $L$, and that $\sigma$ is represented by a charge 
$k$ on $L$. Then, according to [19, Chapter X, Section 5.4], we have that

$$
q_{M, \sigma}\left(\left[m_{j}\right]\right)=\frac{1}{2}-\frac{k_{j}}{2 b_{j j}} \bmod 1 .
$$

Substituting $k_{j}=1-s_{j}+\sum_{i} b_{i j}$, we find that this formula agrees with (2.5) of Example 2.2. This proves the Theorem in this particular case. Now consider the general case, when $L$ is not necessarily algebraically split. We shall use the following observation due to Ohtsuki.

Lemma 3.3 Let $M$ be an oriented rational homology 3-sphere. There exist non-zero integers $n_{1}, \ldots, n_{r}$ such that $M \# L\left(n_{1}, 1\right) \# \cdots \# L\left(n_{r}, 1\right)$ can be presented by surgery along a framed link $L$ algebraically split in $\mathbf{S}^{3}$.

Here \# denotes connected sum and $L(n, 1)$ is the 3 -dimensional lens space obtained by surgery along a trivial knot with framing $n \neq 0$ in $\mathbf{S}^{3}$. Apply that lemma to the oriented rational homology 3 -sphere $M$ we are working with, and consider the resulting manifold $M^{\prime}=M \# L\left(n_{1}, 1\right) \# \cdots \# L\left(n_{r}, 1\right)$. Set $\sigma^{\prime}=\sigma \# \sigma_{1} \# \cdots \# \sigma_{r} \in \operatorname{Spin}^{c}\left(M^{\prime}\right)$ where $\sigma_{1}, \ldots, \sigma_{r}$ denote arbitrary $\operatorname{Spin}^{c}{ }_{-}$ structures on the lens spaces. Then, we have $q_{M^{\prime}, \sigma^{\prime}}=\phi_{M^{\prime}, \sigma^{\prime}}$. By definition of \#, there is a small 3-ball $B \subset M$ such that $M \backslash B \subset M^{\prime}$. This inclusion induces a (injective) homomorphism $i_{*}: H_{1}(M) \rightarrow H_{1}\left(M^{\prime}\right)$. Since we can compute $\phi_{M^{\prime}, \sigma^{\prime}}$ from a split surgery presentation of $M^{\prime}$ using the surgery formula (2.4), we have that $\phi_{M, \sigma}=\phi_{M^{\prime}, \sigma^{\prime}} \circ i_{*}$. It follows from [19, Chapter XII, Section 1.2] (which describes the behaviour of the Reidemeister-Turaev torsion under \#) that, similarly, $q_{M, \sigma}=q_{M^{\prime}, \sigma^{\prime}} \circ i_{*}$. We deduce that $q_{M, \sigma}=\phi_{M, \sigma}$ and we are done.

\section{References}

[1] M F Atiyah, Riemann surfaces and spin structures, Ann. Sci. Ec. Norm. Supér. IV Sér. 4 (1971) 47-62

[2] F Deloup, On Abelian quantum invariants of links in 3-manifolds, Math. Ann. 319 (2001) 759-795

[3] F Deloup, G Massuyeau, Quadratic functions and complex spin structures on three-manifolds, preprint (2002) arXiv:math.GT/0207188

[4] C Gille, Sur certains invariants récents en topologie de dimension 3, Thèse de Doctorat, Université de Nantes (1998)

[5] F Hirzebruch, A Riemann-Roch theorem for differentiable manifolds, Séminaire Bourbaki 177 (1959) 
[6] D Johnson, Spin structures and quadratic forms on surfaces, J. London Math. Soc. 22 (1980) 365-373

[7] R C Kirby, The topology of 4-manifolds, LNM 1374, Springer (1981)

[8] B Lawson, M-L Michelson, Spin geometry, Princeton Univ. Press (1989)

[9] C Lescop, Global surgery formula for the Casson-Walker invariant, Annals of Math. Studies 140, Princeton Univ. Press (1996)

[10] E Looijenga, J Wahl, Quadratic functions and smoothing surface singularities, Topology 25 (1986) 261-291

[11] L Nicolaescu, The Reidemeister torsion of 3-manifolds, Studies in Math. 30, De Gruyter (2003)

[12] H Seifert, W Threlfall, Textbook of topology, Acad. Press (1980), english translation from the original (1934)

[13] V G Turaev, Reidemeister torsion and the Alexander polynomial, Math. Sb. 101 (1976) 252-270

[14] V G Turaev, Torsion invariants of 3-manifolds, PIMS Distinguished Chair Lectures, co-edited by J. Bryden F. Deloup and P. Zvengrowski, University of Calgary (2001)

[15] V G Turaev, Euler structures, nonsingular vector fields, and torsions of Reidemeister type, Iszvestia Ac. Sci. USSR 53:3 (1989), english translation in Math. USSR Izvestia 34:3 (1990) 627-662

[16] V G Turaev, Torsion invariants of Spin ${ }^{c}$-structures on 3-manifolds, Math. Res. Letters 4 (1997) 679-695

[17] V G Turaev, Introduction to combinatorial torsions, Lectures in Math. Series, Birkhäuser (2001)

[18] V G Turaev, Surgery formula for torsions and Seiberg-Witten invariants of 3-manifolds, preprint (2001) arXiv:math.GT/0101108

[19] V G Turaev, Torsions of 3-dimensional manifolds, Progress in Math. 208, Birkhäuser (2002) 